\title{
Integration of Isan Traditional Knowledge in the Holistic Health Treatment of Postpartum Mothers
}

\author{
Chontira Rubngam ${ }^{1}$, Kosit Paengsroy ${ }^{1} \&$ Taksina Krairach ${ }^{1}$ \\ 1 The Faculty of Cultural Science, Mahasarakham University, Khamriang Sub-District, Kantarawichai District, \\ Maha Sarakham Province, Thailand \\ Correspondence: Chontira Rubngam, 73/15 Kasetsomboon Road, Kalasin Sub-district, Mueang District, Kalasin \\ Province 46000, Thailand. E-mail: crubngam126@gmail.com
}

Received: June 3, 2014 Accepted: June 26, 2014 Online Published: June 26, 2014

doi:10.5539/ach.v6n2p227 URL: http://dx.doi.org/10.5539/ach.v6n2p227

\begin{abstract}
This is a qualitative cultural research into the traditional knowledge used by Northeastern Thai communities to care for mothers during the postpartum period. The research objectives were to study the background, current conditions and problems with the integration of Isan traditional knowledge in the holistic health treatment of postpartum mothers in order to outline an overview of the integration process. Following investigation into nine communities in Northeastern Thailand, it was found that three prominent forms of treatment are adopted. These are namely yu fai, tab maw gleau and herbal massage therapy. The traditional medicine has suffered in recent times through lack of inheritance and changing social conditions causing mothers to seek convenient treatment at local hospitals. There are complaints from these new generation mothers about aches, pains and tiredness that were rarely heard in the past. Thus integration of ancestral wisdom in holistic health care provides a perfect solution to the need for recuperation and the fast recovery of the postpartum mother so that she may take care of her child and return to work.
\end{abstract}

Keywords: traditional knowledge, Isan, health care, holistic, postpartum, mother, integration

\section{Introduction}

Society is a group of people that share the same lifestyle, relationships and actions. There are shared and planned existence, customs, culture and values. Sociology reveals the birth, existence, adaptation and inheritance of society. Social change is a dynamic and frequent phenomenon that influences the people within its structures. Everywhere, family is the smallest social group, yet the most important. The members of the family have a close and tight connection, mutual love and live together from birth to death. Family has a responsibility to take care of babies and children to supplement society with new members. Family is the first group that instructs behavioral and personality characteristics. The family is the place where culture is conserved and inherited from one generation to the next. Additionally, if there are problems or obstacles, the family is the group that provides assistance (Pupaiboon, 2008).

Following the first national economic and social development plan, the make-up of families began to change. Increasingly, people began to move away from their family home to seek work. The family structure gradually became more nuclear and children started to lack the warmth they would receive from the traditional extended family. Additionally, there were higher circumstances of broken families, which had an effect on society (Mookrod, 2009).

Isan traditional knowledge is a collection of processes that have arisen from the effects of geography, history and cultural process mixed with knowledge and experimentation. Isan traditional knowledge is divided into prevention and cure and uses natural, supernatural and spiritual methods. Isan people believe in spirits, of which Pi Taen and Pi Fa Paya Taen are the most powerful in creating earth, water, wind, fire, world and humanity. The nearest spirits to home are $P i B u T a$, who are spirits of dead ancestors that come to take care of their descendants. The Isan people will create small shrines for the spirits in the holy forests (called $B a B u T a$ ) near their homes. Aside from this, Isan people also believe in the presence of spirits and there are ceremonies to call the spirits to provide energy and assistance in certain matters and with certain illnesses.

In the past, there was great emphasis placed upon the care of postpartum mothers and newborns through local 
wisdom and traditional medicine, using local herbs and techniques. With the advent of western medicine and the rise of its popularity in Thailand, these traditional methods have declined in popularity (Damrongwattana, 2007). Thai traditional knowledge is related to the cultural context that has been sequentially developed and is of huge assistance to healthcare. There are many areas of Thai wisdom, which include food, knowledge and healthcare. The area of healthcare is subdivided into many further categories, such as Thai massage, the use of traditional herbs in food and the use of traditional herbs as medicine. One further aspect of traditional wisdom is the use of prayer. However, nowadays there have been medical developments in various fields related to natal care, including pregnancy, labor, postpartum and nursing. There has been particular emphasis on mental and physical care during the postpartum period, as it is the time when the body makes its most rapid transition and has a direct influence on the mind and physique.

Social health care in Thailand is based on Thai culture, which is a method of health care known as health culture. Thai traditional knowledge has had an important and large role in health care from the ancient past and is now a part of everyday life. Traditional knowledge comes from the local awareness of the surrounding environment, trial and error and inheritance of knowledge from the past. The care of postpartum mothers is one further aspect of health culture that has been developed from the wisdom of ancient Thailand and its neighboring countries. The postpartum period is the period after labor for about six to eight weeks. This period is divided into the first high-risk twenty-four hours after labor and the period from twenty-four hours to six to eight weeks after labor. Local wisdom includes the belief that heat cures tiredness, improves body temperature and blood circulation. There is also nutritional advice. The traditional knowledge aims to return the body to its original state, with the exception of the breasts and mental attitude to motherhood. It is thus necessary for the mother to receive help from the family so that she may smoothly pass through the initial six to eight weeks after labor (Puabradeut, 2009).

There are a number of areas in which postpartum mothers must take special care and have special understanding, especially first-time mothers who do not yet have the experience of rearing a child. Particularly important are care of the body, cleanliness and personal hygiene, nursing method, nutrition, care of the newborn and mental care and awareness. One further thing to be aware of is the relationship between the mother and her husband or partner, which may be affected by the presence of a new family member. These are changes that the mother must be aware of and prepared for.

In its research into the sanitation of mothers and children, The Thai Ministry of Public Health has identified a number of problems in children aged 0-5 years old. These include iodine deficiency and anemia in pregnant mothers, high pregnancy rates for women under twenty years of age, oxygen deficiency in newborns, underweight newborns, HIV transmission from mother to child, nursing for less than six months and many cases of tooth decay. There is thus a need to standardize health care and improve the quality of care in these areas (Ministry of Public Health, 2011).

There are four primary problems with the care of postpartum mothers. These problems are: a) social change driven by the spread of Western concepts and technology; b) the changing responsibilities of women in the family; c) the decreased role of local traditional knowledge; d) the traditional Thai medical practitioners have an increased role and although the care of postpartum mothers has not vanished from the community, there is no real education of knowledge surrounding the choices and benefits of Thai traditional medicine for the postpartum mother.

For the reasons discussed above, the researchers saw and realized the importance of integrating local Isan traditional knowledge in the holistic healthcare of postpartum mothers. This is a cultural innovation that utilizes the rich and valuable heritage of the society to nurture postpartum mothers back to normal pre-pregnancy health. This is a response to the lifestyle needs of the Isan people, yet there remain the questions of how and if this integration can be achieved. Thus, the researchers were interested in supporting and caring for postpartum mothers in order to reap rewards from the local Isan traditional knowledge and provide future benefits for society.

\section{Research Methodology}

\subsection{Research Aims}

This research, integration of Isan traditional knowledge in the holistic health treatment of postpartum mothers, had three primary research aims, which were: a) to study the background of holistic health treatment of postpartum mothers in the Isan region; b) to study the current conditions of and problems with integration of local Isan traditional knowledge in the holistic health treatment of postpartum mothers; c) to study integration of local Isan traditional knowledge in the holistic health treatment of postpartum mothers. 


\subsection{Research Content}

The content of this research was chosen in line with the three research aims. Regarding the first aim to study the background of holistic health treatment of postpartum mothers in the Isan region, the content included the background and importance of holistic health care for postpartum mothers in the Isan region, as well as the beliefs of mothers concerning holistic health care in the Isan region. Regarding the second aim to study current conditions of and problems with integration of local Isan traditional knowledge, the content covered the conditions and problems of both using traditional knowledge and implementing healthcare for postpartum mothers in the Isan region. Regarding the third and final aim of studying integration for the use of local Isan traditional knowledge in the holistic health treatment of postpartum mothers, the content detailed the integration, development and sponsorship of using local Isan traditional knowledge in the holistic health treatment of postpartum mothers. This research began in September 2012 and was concluded in July 2013.

\subsection{Research Area}

For this investigation, nine communities from three different provinces were chosen to make up the research area. These communities were: a) Moo 9, Akka Village in Khlong Kham Sub-District, Yang Talat District, Kalasin Province; b) Moo 11, Sawang Arom Village in Yang Talat Sub-District, Yang Talat District, Kalasin Province; c) Moo 18, Thai Jaroen Village in Yang Talat Sub-District, Yang Talat District, Kalasin Province; d) Moo 1, Piya Village in Chonnabot Sub-District, Chonnabot District, Khon Kaen Province; e) Moo 2, Piya Village in Chonnabot Sub-District, Chonnabot District, Khon Kaen Province; f) Moo 3, Piya Village in Chonnabot Sub-District, Chonnabot District, Khon Kaen Province; g) Moo 1, Don Som Boy Village in Na Loeng Sub-District, Muang Sam Sip District, Ubon Ratchatani Province; h) Moo 5, Nongbua Village in Na Loeng Sub-District, Muang Sam Sip District, Ubon Ratchatani Province; i) Moo 8, Tungmanee Village in Na Loeng Sub-District, Muang Sam Sip District, Ubon Ratchatani Province.

\subsection{Research Population}

The population for this research was selected using a purposive sampling method. The sample was divided into three groups: key informants, casual informants and general informants. The sixty-strong key informant group was comprised of six government officials, nine local practitioners, eighteen local community leaders, eighteen community health volunteers and nine community elders. There were thirty three casual informants, including six government public health workers, nine Thai herb practitioners and eighteen postpartum mothers. The final group of general informants consisted of eighteen postpartum husbands and eighteen postpartum family members, totaling thirty six individuals. There was a total of one hundred and twenty nine informants for this study.

\subsection{Research Methodology}

This is a cultural qualitative research and used both documentary study and field research. The primary research tools were basic survey, observation, interview and focus group discussion. For the observation, both participant and non-participant observation was performed. For the interview, both structured and non-structured interviews were conducted with all three informant groups. The information was collected and sorted according to the research aims. The information was validated using a triangulation method. Data was then analyzed by using method of agreement, typological analysis and according to the objectives. Data was considered in line with related theories, using the techniques outlined by Songkoon Chantachon (2007). The research results are here presented as a descriptive analysis.

\section{Results}

\subsection{Background of Holistic Health Treatment of Postpartum Mothers in the Isan Region}

Isan is a large area that covers a third of the territory of Thailand and encompasses twenty provinces. The size of Isan means that it is home to a large variety of ethnic groups with different cultures, customs and traditions. Over the years, these traditions have been adopted and adapted across the region to form a general Isan culture that, although has regional variations, is recognizable as a part of the Isan identity. The general background of the nine communities selected for the research is outlined in Table 1. 
Table 1. General background of the nine communities selected for the research

\begin{tabular}{|c|}
\hline $\begin{array}{l}\text { Moo 9, Akka Village in Khlong Kham Sub-District, Yang Talat District } \\
\text { Moo 11, Sawang Arom Village in Yang Talat Sub-District, Yang Talat District } \\
\text { Moo 18, Thai Jaroen Village in Yang Talat Sub-District, Yang Talat District } \\
\text { - } \quad \text { Large communities with many residents } \\
\text { - } \quad \text { Belief in the Heet 12, Kong } 14 \text { system } \\
\text { - } \quad \text { Belief in spirits and the care of spirits, ancestral spirits and mediums } \\
\text { - Local agricultural wisdom includes the planting of organic vegetables, herbs, brown rice for health } \\
\text { - } \text { hocal traditional medicine includes the use of herbs against diabetes and osteoarthritis, to relieve pressure, } \\
\text { - There is education from small and large early learning development centers (primary schools) } \\
\text { There is a large general district hospital which is far and inconvenient to reach, so there is a variety of local } \\
\text { spirit customs to compensate, including pi na, pi ba and pi baan. These customs are aimed to improve the } \\
\text { health of the sick and require the use of herbs by practitioners or local elders with experience. }\end{array}$ \\
\hline $\begin{array}{l}\text { Moo 1, Piya Village in Chonnabot Sub-District, Chonnabot District } \\
\text { Moo 2, Piya Village in Chonnabot Sub-District, Chonnabot District } \\
\text { Moo 3, Piya Village in Chonnabot Sub-District, Chonnabot District } \\
\text { - } \quad \text { There is one temple in the community, Wat Mongkon Luang. There is belief in the local spirit of Pra Jao Yai } \\
\text { Peubang and an annual ceremony has been held on the full moon of the fifth month since } 1949 . \\
\text { - } \quad \text { Belief in spirits and the care of spirits, ancestral spirits and mediums } \\
\text { - } \quad \text { Aglief in the Heet 12, Kong } 14 \text { system } \\
\text { - } \quad \text { Local traditional medicine includes the use of herbs against diabetes and the use of herbal alcohol or boiled } \\
\text { medicine } \\
\text { - There is one primary school in the area } \\
\text { There is a large general district hospital which is far and inconvenient to reach, so there is a need for care } \\
\text { within the community by practitioners or local elders with experience. }\end{array}$ \\
\hline
\end{tabular}

\section{Ubon Ratchatani Province}

Moo 1, Don Som Boy Village in Na Loeng Sub-District, Muang Sam Sip District

Moo 5, Nongbua Village in Na Loeng Sub-District, Muang Sam Sip District

Moo 8, Tungmanee Village in Na Loeng Sub-District, Muang Sam Sip District

- Buddhist traditions and customs

- Belief in spirits and the care of spirits, ancestral spirits and mediums

- Wedding, ordination and bung fai ceremonies

- Belief in the Heet 12, Kong 14 system

- Local agricultural wisdom includes the planting of organic vegetables, herbs, brown rice for health

- Local traditional medicine includes the use of herbs against diabetes, herbal massage and herbal body heating against osteoarthritis, diabetes, pressure, respiratory disease, poisoning and heart disease.

- There is a local school for primary and lower secondary education

- There is a sub-district health center (hospital) but, for many people, transportation to the local hospital is inconvenient so there has been development over time of many alternative therapies to sickness and disease.

There are a number of different techniques adopted by traditional practitioners in the holistic health treatment of postpartum mothers in the three provinces covered by this research. These techniques all use recognized local traditional knowledge. Yu Fai is the practice of sleeping near heat. It is believed that the heat will allow the mother's body to return to its normal pre-pregnancy state, boost the production of breast milk and maintain the mother's health. This ancient practice is beginning to change with the new generation of mothers, who consider convenience above all else. The most common style of $y u$ fai is with the female lying on a narrow stretcher or wooden board. The mother must lay still so as not to roll off the stretcher. To the side, balefires or coal stoves will be started, usually with specially selected fuel so as not to create too much or too poisonous smoke. An alternative method is to place the fire under the stretcher. The mother will spend time in these conditions, depending on local culture, and may not leave the heated room at any time for fear of sickness or disease, even 
taking their newborn with them in a specially crafted basket. Aside from this, the mother will also take hot showers and only drink warm water during the postpartum period. Postpartum mothers are advised by traditional knowledge to only eat certain foods, especially foods high in salt content as it is believed that these will allow the mother to compensate for and replace the salt that was lost through sweating during the $y u$ fai procedure. $Y u$ fai has been accepted and adopted as a beneficial practice across Thailand, although the details of the implementation may differ by location. The husband and family must take responsibility for caring for the mother during the $y u$ fai procedure.

Tab maw gluea is a technique derived from ancient wisdom whereby salt and herbs are baked in a clay pot. The pot is then wrapped in leaves and cloth and the bundle is dabbed on the body. The heat will help open the pores and allow the goodness of the herbs to pass to the skin, help the circulation and help the womb heal. The procedure can also cure tiredness and aches. In the past, this technique was used solely for postpartum mothers but nowadays it is used by people wishing to lose weight and improve the quality of their skin. The procedure can be used on postpartum mothers twelve days after labor but for women having undergone a cesarean section, they must wait until the wound is dry. It is important to ensure that the pot is not too hot. Care must be taken when the pot is used around the breast area as it may cause tightening of the chest. The procedure is best performed in the morning and must not be done while the mother is ill. Tab maw gluea uses herbs such as plai, wild turmeric and camphor.

Herbal massage is another technique that is used on postpartum mothers in their recovery. This has been increasingly accepted by society and has an increased role in local medicine. There are professional standards and laws relating to the practice of Thai herbal massage. The massage techniques derived from traditional knowledge surrounding weight problems among postpartum mothers. Herbs are used in massage of the stomach area to maintain the quality of the skin and reduce fat. The massage is aimed at returning the physical condition of the body to its pre-pregnancy state and cure aches and pains. There are many types of massage used on postpartum mothers, including nuad kao dakiyeb. The masseurs must have experience and expertise in massage technique and the incorporation of herbs in the procedure.

\subsection{The Current Conditions of and Problems with Integration of Local Isan Traditional Knowledge in the Holistic Health Treatment of Postpartum Mothers}

The current conditions of and problems with the integration of local Isan traditional knowledge in the holistic health treatment of postpartum mothers is outlined in Table 2.

Table 2. The current conditions of and problems with the integration of local Isan traditional knowledge in the holistic health treatment of postpartum mothers

\section{Yu Fai \\ Current Conditions}

$Y u f a i$ is the responsibility of nurses and there must always be someone caring for the patient. The process allows for the rapid recovery of the labor wounds, reshaping of the stomach, return of health and sufficient production of breast milk.

\section{Problems}

It was not effectively inherited by the future generations as an alternative method of treatment and thus the practices are not well known. If the mothers do not eat the correct diet, breast milk production will be insufficient.

\section{Tab Maw Gluea \\ Current Conditions}

This is one aspect of ancestral wisdom that has been inherited from the past to take care of postpartum mothers so that the health of the mother is in good conditions and their physique returns to the pre-pregnancy stage. There are specific procedures that must be performed and there is a lot of herb mixing. It is important that the sick person must not perform the procedure themselves. This must not be performed within seven days of labor, if the mother has a temperature over 37.5 degrees Celsius or if they have a full stomach.

\section{Problems}

Care must be taken to ensure that the pot is not too hot. The procedure must not be performed if the mother is sick or if they have just had a cesarean section. The procedure must be performed after the womb has returned to its normal state, ideally two weeks after labor. For women who have undertaken a cesarean section, they must wait for a month before performing tab maw gluea. There are many steps to the procedure and a number of things to be careful of: a) do not perform if pregnant; b) there must not be strong air currents or cool air 
conditioning; c) the mother must rest flat after the procedure; d) the procedure should not be performed on a full stomach and the mother must go to toilet before the procedure; e) the procedure must not be performed if the mother as a stomach ache, sickness or if there is internal injury; f) if the procedure is performed around the xiphoid process (lower sternum), the edges of the ribcage, the pelvis, hips, vulva or spinal column, there may be bruising or swelling.

\section{Herbal Massage \\ Current Conditions}

Herbal massage is a method to improve blood circulation and remove toxins via the kidney and urine. This is also a way of maintaining health of postpartum mothers, increasing skin quality and removing fat. Herbs used include plai, lemongrass, curcuma zedoria roscoe, turmeric and kaffir lime skin, among others. These herbs are combined in a sachet and steamed until they fuse. This sachet is then applied to the massage area (invariably over a cloth so as not to burn the skin underneath).

\section{Problems}

Beneficial herbs must be used. Roots and heartwood must not be used. Postpartum mothers often encounter problems of strains and allover body fatigue. This causes a lack of self-confidence in the ability of the postpartum mother to maintain their own health and condition. Traditional herbal massage is the best folk-wisdom-inspired way to remedy these problems.

\subsection{The Integration of Local Isan Traditional Knowledge in the Holistic Health Treatment of Postpartum Mothers}

Nowadays, the majority of mothers give birth at health centers, such as hospitals. After birth, they will be given a course of medication to relieve pain and symptoms of illness. Therefore, they do not place as much importance on the yu fai procedure as they should. However, modern mothers often complain of aches, pains, tiredness, illness and insufficient breast milk production, which could be remedied by the $y u$ fai procedure. With altering roles of women in society and changes to the economy, mothers are expected to work more and have less time for traditional treatments. Also, improvements in transport have made the hospitals more accessible. There are also less people to take care of the postpartum mother, so the $y u$ fai procedure is neglected. The existing $y u$ fai procedures have thus been adapted to be more suitable for modern society and more appropriate for a working family.

Tab maw gluea is another traditional heat treatment that has been passed on from generation to generation. The practice of heating salt and herbs in a clay pot before using it to treat the body aids circulation, respiration, lochia discharge, recovery from aches and pains and return of physical normality and positioning of the womb. The procedure should be performed twice a day for one or two hours, ideally in the early morning and the afternoon. Nowadays the procedure can be performed in local hospitals and health centers.

The recovery of postpartum mothers using traditional herbal massage as a form of treatment is rapid and successful, helping them recover from all manner of complaints. The massage techniques are similar to general massage, although not as firm and strong and herbs are used to supplement the massage. The period after labor is a time during which the mother must rest and relax to recover from the body changes during pregnancy and labor, particular the fluid that was lost during and after labor. The wisdom of ancestral generations regarding herbal massage provides a perfect solution to this need for recuperation and the fast recovery of the mother so that she may take care of her child and return to work.

\section{Discussion}

The integration of local Isan traditional knowledge in the holistic health treatment of postpartum mothers relies on the experience of community locals (often elders) and specialist postpartum care-givers in the yu fai, tab maw gluea and herbal massage treatments and their understanding, use and combining of the knowledge (especially knowledge of herbs) they have received from their ancestors, so that it is appropriate for modern society. Alongside this ancient wisdom are the development, integration and adaptation of new methods. Thus a new body of knowledge is created that is appropriate for modern society and modern environmental conditions. This is in agreement with the symbolic interaction theory, which states that for society to occur there must be human interaction. This social interaction involves the local wisdom of the people, culture, customs, traditions and lifestyle. These societies use and integrate existing wisdom to provide social care.

This research is related to the holistic health care theory in that it advocates treatment of physical, emotional, spiritual, social and environmental ills. The people in the community must have the self-awareness and the ability to care for themselves by adopting a healthy lifestyle. When practitioners have identified an illness, the 
patient must take full responsibility to ensure they get the appropriate treatment. One such example for postpartum mothers is diet control. Society must outline recommendations and warnings by research through interview and survey. The findings should then be used to create a wellness education with the objectives of raising health awareness in the community and enabling locals to take care of themselves.

The use of Thai traditional medicine to aid the recovery of postpartum mothers agrees with the policies of the fifth national economic and social development plan, as well as modern concepts of childcare that outline desired practice for the rearing of children. These theories, which include nursing for at least six months, proper dietary planning and the building of relationships between father, mother and child, correspond to the structural functionalism theory. This states that social needs are necessary for mental stability, as they create warmth and confidence in the self. They require a social model or role-model, which leads to a relationship with the cultural diffusion theory (Pongsapit, 2006). The cultural diffusion theory concludes that different societies have similar cultures as a result of cultural diffusion from one to another. This means that the traditional knowledge related to postpartum mothers that is adopted and integrated in health care across Isan and beyond first stemmed from cultural diffusion between its ethnic societies. This is in agreement with the research of Daranee Onchomjan (2009), who stated that the problems with holistic health care do not lie with the integrated ancient knowledge but rather with the understanding and application of that knowledge in terms of culture, customs, traditions, religion and beliefs. This research also corresponds to the theories of Dujduean Samranwong (2001), who stated that care must be balanced between activity and rest, between private time and public time. Concerning remedies that use traditional knowledge and that are suitable for beliefs and culture in Isan, this research agrees with the findings of Mara Chaiyanek, whose study of Phu Tai people in Kalasin Province found that yu fai and traditional herbal remedies were used to care for postpartum mothers (Chaiyanek, 2001).

In the past, modern medicine and technology in Isan was not widespread, so the majority of expecting mothers were not able to use modern methods of treatment and the traditional knowledge alternatives were preferable. Nowadays, this treatment is known as alternative medicine and refers to the adaptation of traditional knowledge of ancient communities in health care. This traditional medicine aims to improve the lifestyle and quality of the life of Isan people, while conserving and passing on traditional knowledge and wisdom to future generations.

\section{References}

Chaiyanek M. (2001). Factors related to the behavior of self-care according to traditional knowledge among postpartum mothers: A case study of the Phu Tai people of Ban Kud Wa, Kuchinarai District, Kalasin Province [in Thai]. Bangkok: Kasetsart University.

Chantachon S. (2007). Advanced cultural qualitative research [in Thai]. Maha Sarakham, Mahasarakham University, documents used for teaching.

Damrongwattana, J. (2007). Study of healthcare for pregnant women, postpartum mothers and newborn children using local traditional knowledge procedures in Nakhon Sri Thammarat [in Thai]. Nakhon Sri Thammarat University: Ministry of Education.

Mookrod, S. (2009). Results of the sponsored nursing system and provision of knowledge for the healthcare behavior of mothers towards their children and themselves [in Thai]. Bangkok: Chulalongkorn University Press.

Ministry of Public Health. (2011). Guidebook for postpartum mothers and the care of newborns [in Thai]. Nonthaburi: Ministry of Public Health.

Onchomjan, D. (2009). Nurturing pregnancies in the past and the care of mother and child in Thai-Muslim communities in five Southern border provinces [in Thai]. Retrieved from http://www.dtam.moph.go.th

Pongsapit, A. (2006). Cultural variety: Paradigm and role in civil society [in Thai]. Bangkok: Chulalongkorn University Press.

Puabradeut, W. (2009). Care of high risk pregnancies [in Thai]. Bangkok: Maw Chao Baan.

Pupaiboon, R. (2008). Concepts, theories and uses of family nursing [in Thai]. Bangkok: V J Printing.

Samranwong, D. (2001). Study of the behavior of self-care among postpartum women [in Thai]. Khon Kaen: Khon Kaen University. 


\section{Copyrights}

Copyright for this article is retained by the author(s), with first publication rights granted to the journal.

This is an open-access article distributed under the terms and conditions of the Creative Commons Attribution license (http://creativecommons.org/licenses/by/3.0/). 